\title{
BMJ Open Stress resilience and the risk of inflammatory bowel disease: a cohort study of men living in Sweden
}

\author{
Carren Melinder, ${ }^{1}$ Ayako Hiyoshi, ${ }^{1}$ Katja Fall, ${ }^{1}$ Jonas Halfvarson, ${ }^{2}$ \\ Scott Montgomery ${ }^{1,3,4}$
}

To cite: Melinder C, Hiyoshi A, Fall K, et al. Stress resilience and the risk of inflammatory bowel disease: a cohort study of men living in Sweden. BMJ Open 2017;7:e014315. doi:10.1136/bmjopen-2016014315

- Prepublication history for this paper is available online. To view these files please visit the journal online (http://dx.doi.org/10.1136/ bmjopen-2016-014315).

Received 19 September 2016 Revised 28 November 2016 Accepted 2 December 2016

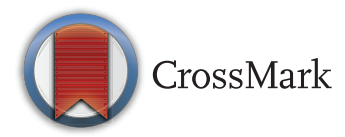

${ }^{1}$ Department of Clinical Epidemiology and Biostatistics, School of Medical Sciences, Örebro University, Örebro, Sweden ${ }^{2}$ Department of

Gastroenterology, School of Medical Sciences, Örebro University, Örebro, Sweden ${ }^{3}$ Department of Epidemiology and Public Health, University College London, London, UK ${ }^{4}$ Clinical Epidemiology Unit, Karolinska University Hospital, Karolinska Institutet, Stockholm, Sweden

Correspondence to Carren Melinder; carren.melinder@oru.se

\section{ABSTRACT}

Objective: To determine if low psychosocial stress resilience in adolescence (increasing chronic stress arousal throughout life) is associated with an increased inflammatory bowel disease (IBD) risk in adulthood. Subclinical Crohn's disease (CD) and ulcerative colitis (UC) can exist over many years and we hypothesise that psychosocial stress may result in conversion to symptomatic disease through its proinflammatory or barrier function effects.

Design: National register-based cohort study of men followed from late adolescence to middle age.

Setting: A general population cohort of men in Sweden.

Participants: Swedish population-based registers provided information on all men born between 1952 and 1956 who underwent mandatory Swedish military conscription assessment ( $\mathrm{n}=239$ 591). Men with any gastrointestinal diagnoses (except appendicitis) prior to follow-up were excluded.

Primary outcome measures: An inpatient or outpatient diagnosis of CD or UC recorded in the Swedish Patient Register (1970-2009).

Results: A total of 938 men received a diagnosis of $C D$ and 1799 UC. Lower stress resilience in adolescence was associated with increased IBD risk, with unadjusted $\mathrm{HRs}$ ( $95 \% \mathrm{Cls}$ ) of 1.54 (1.26 to 1.88) and 1.24 (1.08 to 1.42), for CD and UC, respectively. After adjustment for potential confounding factors, including markers of subclinical disease activity in adolescence, they are 1.39 (1.13 to 1.71) and 1.19 (1.03 to 1.37).

Conclusions: Lower stress resilience may increase the risk of diagnosis of IBD in adulthood, possibly through an influence on inflammation or barrier function.

\section{INTRODUCTION}

The aetiology of inflammatory bowel disease (IBD) - Crohn's disease (CD) and ulcerative colitis (UC) - is believed to involve an interaction between genetic and environmental factors that results in an atypical immune response to gut microbiota. ${ }^{1-3}$

\section{Strengths and limitations of this study}

- This study used a prospectively collected measure of stress resilience (susceptibility to stress) in adolescence to examine its association with the risk of subsequent Crohn's disease (CD) and ulcerative colitis (UC) in adulthood.

- Stress resilience was measured before typical inflammatory bowel disease (IBD) onset age, and potential effects of prodromal disease activity in adolescence were taken into account in the analysis.

- Since we examined only stress resilience and did not have a direct measure of coping to capture the extent to which situations were perceived as stressful, we may have underestimated the magnitude of associations between stress and IBD.

- The study did not have a measure of smoking and while this could be a pathway relevant to $\mathrm{CD}$, it cannot explain the increased risk of UC.

- The results are based on men up to middle age and may not be applicable to older ages or to women.

Genetic factors are clearly important in the aetiology, ${ }^{14}$ but the importance of environmental factors is signalled by temporal trends in incidence. ${ }^{5}$ It appears that exposures in early life are potentially relevant to bowel colonisation and homoeostasis, ${ }^{6}$ such as infections and antibiotic therapy, ${ }^{7}$ are particularly important in determining lifetime IBD risk suggesting a long and silent natural history $^{8}$ as frank disease onset occurs in adulthood. ${ }^{9}$ Acute appendicitis before age 20 years has been linked consistently with a reduced risk of $\mathrm{UC}^{10}{ }^{11}$ and it has been suggested recently that this may be due to genetic factors influencing risk of appendicitis and UC risk. ${ }^{11}$ Some exposures in adulthood may be risks, ${ }^{5}$ but the best documented risk is cigarette smoking, which is associated with an increased risk of CD, but a reduced risk of UC..$^{5}$ 
Psychosocial stress may increase inflammation, including through sympathetic nervous system influences, ${ }^{12}$ and as subclinical low-grade inflammation related to IBD can exist over many years, ${ }^{8}$ it is possible that stress may result in conversion to symptomatic IBD. Inflammation can increase permeability and compromise the integrity of the gastrointestinal mucosal barrier; thus, stress may promote passage over the epithelial barrier of bacterial pathogens and activate mucosal immune responses. ${ }^{13}$

Studies of psychosocial stress and IBD exacerbations have generated inconsistent results. ${ }^{14-17}$ Low levels of stress and better coping strategies are associated with reduced risk of $\mathrm{CD}$ and $\mathrm{UC}$ relapses in prospective studies $^{14}{ }^{15}$ but not life events. ${ }^{16}{ }^{17}$ Psychosocial stress was associated with an increased risk of CD but not UC in a prospective cohort study ${ }^{18}$ but not in other studies. ${ }^{19} 20$ To the best of our knowledge, no previous study has considered individual variation in stress susceptibility, a potentially important determinant of chronic stress arousal. ${ }^{21}$ Here we use Swedish register data to examine the association of stress resilience in adolescence with $\mathrm{CD}$ and $\mathrm{UC}$ risk in subsequent adulthood.

\section{MATERIALS AND METHODS}

Study population

The study population and measures have been described in detail elsewhere. ${ }^{22}{ }^{23}$ Briefly, the participants were men born during 1952-1956. Most men were 1819 years of age when they attended compulsory Swedish military conscription assessments during 1969-1976. The follow-up period for CD and UC was from 4 years after the conscription assessment (to reduce the possibility of reverse causation, such that symptomatic disease reduced stress resilience), until diagnosis, migration, death or end of the study, 31 December 2009 (to a maximum age of 57 years).

The entire cohort comprised 284198 men. Exclusions were for female sex, uncertain vital status or personal number, emigration or death before follow-up ( $n=5504)$. Men who emigrated or died before the follow-up period were excluded because they could not contribute to the results. We also excluded men who were assessed before 17 years of age, did not undertake part of the conscription assessments or had missing data $(n=35101)$. Additionally, cohort members were excluded if they had any gastrointestinal diagnoses (except appendicitis), including IBD, at the conscription assessment including when recorded in the Patient Register prior to follow-up $(n=4002)$. In total, $44607(15.7 \%)$ men were excluded. The majority of the exclusions (11.7\% of the cohort) were due to missing data for variables used in the analysis.

\section{Data sources and measures}

Socioeconomic and demographic data

Parental socioeconomic index (SEI) during childhood was obtained using the Population and Housing Census in 1960. We classified parental occupation into business owners/managers, farm owner/managers, manual workers, agricultural workers, office workers and other. Data on date of birth, sex, region of residence, vital status (dead or alive) and migration were obtained from the Total Population Register.

\section{The Swedish Military Service Conscription Register}

Military service was compulsory for all men from age 18 years, with exceptions including those with severe medical conditions, and entry involved a detailed assessment. $^{23}$

\section{Stress resilience}

Examination of psychological function at the conscription assessment produced a stress resilience score from 1 to 9, categorised as low (1-3), medium (4-6) and high (7-9), to maintain consistency with previous studies. ${ }^{24-26}$ Following completion of a questionnaire, the interview was carried out by psychologists, whose inter-rater reliability was evaluated on a regular basis ${ }^{27}$ and estimated to be high $(\mathrm{r}=0.85)$ by a study conducted in 1972 and $1973 .^{28}$ The interview, which usually took $20-30 \mathrm{~min}$, explored the experience of potential conflicts or adjustment problems, as well as the ability to take the initiative and assume responsibilities at school, work or home. ${ }^{28}$ The measure is based on five-scale or nine-scale normally distributed ratings of psychological energy, emotional control and social maturity. ${ }^{26-29}$ Emotional control evaluated the ability to tolerate psychological stress in general as well as mental stability and emotional maturity. ${ }^{26-29}$ Social maturity assessed if individuals were independent, socially extrovert and responsible. ${ }^{26} 27 \quad 29$ Psychological energy assessed the ability to engage in various activities even when facing adversity. ${ }^{26} 27$

\section{Height and body mass index}

Height in centimetres was divided into fifths of the distribution. Height $<144 \mathrm{~cm}$, weight $>178 \mathrm{~kg}$ or body mass index (BMI) $<15 \mathrm{~kg} / \mathrm{m}^{2}$ was treated as non-valid values and excluded. BMI was calculated from measures of height and weight and categorised as underweight (15$\left.18.49 \mathrm{~kg} / \mathrm{m}^{2}\right)$, normal weight $\left(18.50-24.99 \mathrm{~kg} / \mathrm{m}^{2}\right)$ and overweight/obese $\left(>25 \mathrm{~kg} / \mathrm{m}^{2}\right)$. Since there were few obese men at the conscription assessment, the obese and overweight categories $\left(\mathrm{BMI} \geq 25 \mathrm{~kg} / \mathrm{m}^{2}\right)$ were combined.

\section{Erythrocyte sedimentation rate}

Erythrocyte sedimentation rate (ESR), indicating systemic inflammation, was standardised for erythrocyte volume fraction (EVF) by adjustment ${ }^{22} 23$ and grouped into five categories: $1,2-6,7-10,11-14$ and $\geq 15 \mathrm{~mm} /$ hour. $\mathrm{ESR}<1$ or $>98 \mathrm{~mm} /$ hour and $\mathrm{EVF}<0.20$ or $>0.75$ were considered as non-valid. 
Gastrointestinal diseases at conscription assessment

Gastrointestinal diagnoses up to the time of the conscription assessment were obtained from the Conscription Register and the National Patient Register (NPR). The codes used are the Swedish version of the International Classification of Diseases (ICD) revision 8 (ICD-8 codes 530-539, 543, 555-558, 560-577). Appendicitis prior to age 20 years was identified (ICD-8 codes 540-542) in the Conscription Register and surgical procedure codes (4510 and 4511) in the NPR. We combined appendectomy and appendicitis prior to age 20 years into a single variable.

\section{Geographical regions}

Sweden was divided into northern, central and southern regions.

\section{The National Patient Register}

IBD diagnoses in adulthood were identified through ICD 8,9 or 10 codes in the NPR. Since 1964, the National Board of Health and Welfare has collected information on inpatient diagnoses and the register achieved complete coverage in 1987. The register expanded to include data on outpatient visits in 2001 and $\sim 99 \%$ of all primary hospital diagnoses are recorded. ${ }^{30}$

\section{IBD diagnoses}

Primary and secondary diagnoses in inpatient and outpatient records were identified: CD (563.00 for ICD-8; 555.x for ICD-9; ICD-10 K50.x), and UC (563.10 for ICD-8; 556.x for ICD-9; ICD-10 K51.x). During the follow-up period, 938 and 1799 men were identified as having diagnoses of $\mathrm{CD}$ and $\mathrm{UC}$, respectively. A total of 438 men had records of CD and UC. The most recent diagnosis was used to define disease phenotype, but the time of the first diagnosis defined disease onset. A total of 286 with UC changed diagnosis to CD and 152 men with $\mathrm{CD}$ changed diagnosis to UC.

\section{Statistical analysis}

The association between stress resilience in adolescence and risk of subsequent IBD in adulthood was evaluated by Cox regression. We examined the proportional hazards assumption graphically, with no indication of violation. Separate models were used for CD and UC, with adjustment for parental SEI in childhood, appendicitis prior to age 20 years, region of residence and markers of potential prodromal disease activity in adolescence (ESR, EVF, height and BMI). We also modelled stress resilience as an ordinal or continuous variable to assess the linear trend of associations with IBD risk for three-category and nine-category measures of stress resilience. We examined whether stress resilience modifies the association of BMI, height and inflammation in adolescence with IBD risk using stratification and interaction testing. Interaction terms for ESR, height and BMI with stress resilience were included in
Cox models, with adjustment for the main effects. Age was used as the underlying time scale and, unless otherwise specified, all measures were modelled as categorical variables.

\section{Sensitivity analyses}

Further analyses assessed if changes in diagnostic accuracy influenced the findings (by the end of the 1970s, it was higher) and to assess whether stress resilience in adolescence is associated with a first IBD diagnosis, even after a minimum of 15 years from assessment, as in a previous study. ${ }^{22}$ Among those who had IBD diagnoses (638 CD and $1469 \mathrm{UC}$ ) during this period (that started 15 years after the conscription assessment), 283 men had both CD and UC diagnosis. As is in the main analysis, the most recent diagnosis was used to define disease phenotype, but the time of the first diagnosis defined disease onset. Men who had IBD diagnosis during the period prior to the start of follow-up were excluded from the analysis $(n=1156)$. We also conducted a separate analysis excluding men more likely to have undiagnosed disease in adolescence, defined as low EVF $(\leq 39)$, elevated ESR $(\geq 15)$ or underweight (BMI 15$\left.18.49 \mathrm{~kg} / \mathrm{m}^{2}\right)$.

SPSS software V.23 and Stata V.13 were used. We considered $\mathrm{p}$ values $<0.05$ and $95 \%$ CIs not including 1.00 as statistically significant.

\section{RESULTS}

\section{Participant characteristics}

The study comprised 239591 men followed from 4 years after the conscription assessment in late adolescence to a maximum of age 57 years (table 1 ).

Men with CD were more likely to have lower stress resilience and raised ESR compared with men with UC and men without IBD. Men with IBD were more likely to have lower parental SEI in childhood compared with men without IBD. Men with UC were less likely to have had an appendectomy or appendicitis prior to age 20 years. The median age of onset was 40 years (range 21-57) for $\mathrm{CD}$ and 47 years (range 22-57) for UC.

\section{Stress resilience in adolescence and subsequent CD in adulthood}

Men with low and moderate stress resilience had an increased risk of subsequent $\mathrm{CD}$ compared with men with high stress resilience (table 2). Modelling the threecategory variable as a linear measure, the HRs $(95 \%$ CIs) for the association of stress resilience with $\mathrm{CD}$ (the average change in $\mathrm{CD}$ risk by one unit change in the three stress resilience categories) are 1.23 (1.11 to 1.35) and 1.17 (1.06 to 1.28), before and after adjustment, respectively. Modelling the nine-category measure as linear produces HRs of 1.09 (1.05 to 1.13) and 1.07 (1.03 to 1.11), respectively. Adjustment for any individual potential confounding factor had little influence on 
Table 1 Baseline characteristics of study participants by IBD diagnosis

\begin{tabular}{|c|c|c|c|}
\hline & $\begin{array}{l}\text { No IBD } \\
\mathrm{n}=236854 \\
\mathrm{n}(\%)\end{array}$ & $\begin{array}{l}C D \\
N=938 \\
n(\%)\end{array}$ & $\begin{array}{l}\text { UC } \\
n=1799 \\
n(\%)\end{array}$ \\
\hline \multicolumn{4}{|l|}{ Stress resilience } \\
\hline Low & $50317(24.0)$ & 225 (23.5) & $423(21.3)$ \\
\hline Moderate & $129457(58.3)$ & $547(55.1)$ & $990(54.7)$ \\
\hline High & $57080(17.7)$ & $166(21.4)$ & $386(24.1)$ \\
\hline Mean (SD) & $5.1(1.9)$ & $4.8(1.8)$ & $4.9(1.9)$ \\
\hline \multicolumn{4}{|l|}{ BMI } \\
\hline Mean (SD) $\left(\mathrm{kg} / \mathrm{m}^{2}\right)$ & $21.2(2.6)$ & $21.0(2.6)$ & $21.0(2.5)$ \\
\hline \multicolumn{4}{|l|}{ ESR in adolescence } \\
\hline Mean (SD) (mm/hour) & $3.4(3.5)$ & $5.1(5.9)$ & $3.9(4.2)$ \\
\hline Median (range) & $2(1-89)$ & $3(1-51)$ & $3(1-55)$ \\
\hline \multicolumn{4}{|l|}{ Height } \\
\hline Mean (SD) (cm) & $178.7(6.4)$ & $178.1(6.4)$ & $178.1(6.5)$ \\
\hline \multicolumn{4}{|l|}{ Parental SEI in 1960} \\
\hline Manual worker & 96493 (40.7) & $423(45.1)$ & 766 (42.6) \\
\hline Agricultural workers & $9046(3.8)$ & $52(5.5)$ & $65(3.6)$ \\
\hline Farm owner/managers & $23396(9.9)$ & $65(6.9)$ & $169(9.4)$ \\
\hline Office workers & 65495 (27.7) & $230(24.5)$ & $468(26)$ \\
\hline Business owners/managers & $25267(10.7)$ & $98(10.5)$ & $182(10.1)$ \\
\hline Others (unknown) & $17157(7.2)$ & $70(7.5)$ & $149(8.3)$ \\
\hline \multicolumn{4}{|l|}{ Appendicitis <20 years } \\
\hline No & 234935 (99.2) & 933 (99.5) & $1796(99.8)$ \\
\hline Yes & $1919(0.8)$ & $5(0.5)$ & $3(0.2)$ \\
\hline \multicolumn{4}{|l|}{ Age at diagnosis (years) } \\
\hline Median (range) & & $40(21-57)$ & $47(22-57)$ \\
\hline
\end{tabular}

Table 2 Stress resilience in adolescence and subsequent Crohn's disease risk in adulthood at least 4 years after the conscription assessment

\begin{tabular}{|c|c|c|c|}
\hline Stress resilience & Events/n & Unadjusted $\mathrm{HR}(95 \% \mathrm{Cl})$ & Adjusted* HR (95\% Cl) \\
\hline \multicolumn{4}{|l|}{ Main analysis } \\
\hline Low & $225 / 50965$ & $1.54(1.26$ to 1.88$)$ & $1.39(1.13$ to 1.71$)$ \\
\hline Moderate & $547 / 130994$ & $1.43(1.20$ to 1.70$)$ & $1.36(1.14$ to 1.62$)$ \\
\hline High & $166 / 57632$ & Reference & Reference \\
\hline \multicolumn{4}{|c|}{ Sensitivity analysis - excluding those with elevated ESR, low EVF and underweight } \\
\hline Low & $115 / 41735$ & $1.53(1.23$ to 1.91$)$ & $1.45(1.16$ to 1.81$)$ \\
\hline Moderate & $301 / 113417$ & $1.34(1.11$ to 1.62$)$ & $1.32(1.09$ to 1.59$)$ \\
\hline High & $101 / 53007$ & Reference & Reference \\
\hline \multicolumn{4}{|c|}{ Sensitivity analysis - follow-up at least 15 years after the conscription assessment } \\
\hline Low & $147 / 49278$ & $1.46(1.15$ to 1.87$)$ & $1.37(1.07$ to 1.75$)$ \\
\hline Moderate & $377 / 127750$ & $1.42(1.16$ to 1.76$)$ & $1.39(1.22$ to 1.71$)$ \\
\hline High & $114 / 55644$ & Reference & Reference \\
\hline
\end{tabular}

${ }^{*}$ Adjusted for BMI, ESR, EVF, height, parental SEI, appendicitis before age 20 and region of residence.

$\mathrm{BMI}$, body mass index; ESR, erythrocyte sedimentation rate; EVF, erythrocyte volume fraction; $\mathrm{n}$, number; SEI, socioeconomic index.

the association of stress resilience with $\mathrm{CD}$, and it was the combined adjustment that had a notable influence (data not shown).

The analysis excluding men more likely to have undiagnosed disease activity in adolescence (table 2) limited the sample to 743 men with $\mathrm{CD}$, and the results are consistent with the main analysis. Also, the association between low stress resilience in adolescence and future risk of $\mathrm{CD}$ remained during the follow-up beginning at least 15 years after the conscription assessment in adolescence (table 2). A total of 638 diagnoses of CD were identified and the median age of onset was 47 years (range 33-57). There was no statistically significant interaction between stress resilience and markers of subclinical disease activity in CD ( $p>0.05$ for all, data not shown). 
Stress resilience in adolescence and subsequent UC in adulthood

Men with low stress resilience have a statistically significant increased risk of UC during follow-up compared with men with high stress resilience (table 3) while moderate stress resilience was associated with a lower magnitude and non-statistically significant increased risk of UC. Modelling the three-category variable as a linear measure produced HRs for the association of stress resilience with UC of 1.11 (1.04 to 1.19) and 1.09 (1.02 to 1.17), before and after adjustment, respectively. Modelling the nine-category measure as linear produced HRs of 1.05 (1.02 to 1.07 ) and 1.04 (1.02 to 1.07), respectively. When potential confounding factors were adjusted for, there was no notable influence on the association of stress resilience with UC for any specific individual factor (data not shown).

The analysis excluding men who were more likely to have undiagnosed disease activity in adolescence (table 3) limited the sample to 1517 men with UC. The results are consistent with the main analysis for low stress resilience. Men with moderate stress resilience also had a statistically significant increased risk of UC, compared with men with high stress resilience. Also, the association between low stress resilience in adolescence and future risk of UC remained during the follow-up beginning at least 15 years after the conscription assessment in adolescence (table 3). A total of 1469 diagnoses of UC were identified and the median age of onset was 48 years (33-57).

The only evidence of effect modification by stress resilience for the association of prodromal disease activity markers for UC was for BMI (table 4). Underweight was only associated with a raised risk of UC in those with high stress resilience ( $p$ for interaction $<0.05$ ), suggesting that more aggressive disease in adolescence reduces the apparent protective influence of high stress resilience.

\section{DISCUSSION}

In this national general population-based study of men, we assessed if stress resilience in adolescence is associated with the risk of a diagnosis of IBD in subsequent adulthood, among individuals without any gastrointestinal diagnoses in adolescence. Low stress resilience was associated with an increased risk of $\mathrm{CD}$ and $\mathrm{UC}$, diagnosed at least 4 years after stress resilience was assessed in adolescence; and the association with $\mathrm{CD}$ was of a somewhat higher magnitude. The increased risk remained after adjustment for potential risk factors and markers of prodromal disease activity, as well as after exclusion of men with evidence of prodromal disease activity in adolescence and after extending the follow-up entry to 15 years after the assessment of stress resilience.

Rather than stressful exposures, we used stress resilience as a marker of individual susceptibility to stress. Psychosocial stress increases inflammatory cytokines including interleukin-6, tumour necrosis factor- $\alpha$ and interferons. Long-term exposure to cytokines may cause impaired negative feedback regulation of the hypothalamic-pituitary-adrenal (HPA) axis, resulting in increased cortisol concentrations. ${ }^{12}$ Psychosocial stress may also impair intestinal barrier integrity and this may enable commensal bacteria to cross the gastrointestinal mucosa and provoke inflammation and disease. ${ }^{13}$ Thus, the mechanisms may involve proinflammatory influence and mucosal immune response or impaired barrier function, but the two potential mechanisms are not mutually exclusive. A proinflammation tendency associated with low stress resilience could be of a systemic nature or specific to some organ systems, such as the gut. Previously, we found no association of stress resilience with multiple sclerosis, ${ }^{25}$ where inflammation is confined to the central nervous system compartment and is thus potentially protected from some forms of systemic inflammation. In contrast, we found that low stress resilience is associated with a raised risk of ischaemic

Table 3 Stress resilience in adolescence and subsequent ulcerative colitis risk in adulthood at least 4 years after the conscription assessment

\begin{tabular}{|c|c|c|c|}
\hline Stress resilience & Events/n & Unadjusted HR (95\% Cl) & Adjusted ${ }^{\star}$ HR (95\% Cl) \\
\hline \multicolumn{4}{|l|}{ Main analysis } \\
\hline Low & $423 / 50965$ & $1.24(1.08$ to 1.42$)$ & $1.19(1.03$ to 1.37$)$ \\
\hline Moderate & $990 / 130994$ & $1.11(0.98$ to 1.24$)$ & 1.08 (0.96 to 1.22$)$ \\
\hline High & $386 / 57632$ & Reference & Reference \\
\hline \multicolumn{4}{|c|}{ Sensitivity analysis - excluding those with elevated ESR, low EVF and underweight } \\
\hline Low & $339 / 41735$ & $1.28(1.10$ to 1.49$)$ & 1.26 (1.08 to 1.47$)$ \\
\hline Moderate & $843 / 113417$ & $1.15(1.02$ to 1.31$)$ & $1.14(1.01$ to 1.30$)$ \\
\hline High & $335 / 53007$ & Reference & Reference \\
\hline \multicolumn{4}{|c|}{ Sensitivity analysis - follow-up at least 15 years after the conscription assessment } \\
\hline Low & $345 / 49278$ & $1.26(1.08$ to 1.46$)$ & $1.22(1.04$ to 1.42$)$ \\
\hline Moderate & $813 / 127750$ & $1.12(0.99$ to 1.28$)$ & $1.11(0.97$ to 1.26$)$ \\
\hline High & $311 / 55644$ & Reference & Reference \\
\hline
\end{tabular}


Table 4 BMI in adolescence and subsequent ulcerative colitis risk in adulthood at least 4 years after the conscription assessment, stratified by stress resilience level

\begin{tabular}{|c|c|c|c|}
\hline Stress resilience level & Events/n & Unadjusted HR (95\% Cl) & Adjusted* HR $(95 \% \mathrm{Cl})$ \\
\hline Low & $423 / 50965$ & & \\
\hline Underweight & & $1.00(0.77$ to 1.29$)$ & 0.99 (0.76 to 1.28$)$ \\
\hline Normal weight & & Reference & Reference \\
\hline Obese/overweight & & $0.79(0.53$ to 1.16$)$ & $0.80(0.54$ to 1.17$)$ \\
\hline Moderate & 990/130994 & & \\
\hline Underweight & & $0.98(0.81$ to 1.19$)$ & $0.98(0.81$ to 1.19$)$ \\
\hline Normal weight & & Reference & Reference \\
\hline Obese/overweight & & $0.62(0.46$ to 0.84$)$ & 0.61 (0.45 to 0.82$)$ \\
\hline High & $386 / 57632$ & & \\
\hline Underweight & & 1.71 (1.24 to 2.36$)$ & 1.72 (1.25 to 2.38$)$ \\
\hline Normal weight & & Reference & Reference \\
\hline Obese/overweight & & $1.02(0.69$ to 1.53$)$ & $1.00(0.67$ to 1.50$)$ \\
\hline
\end{tabular}

stroke $^{24}$ and coronary heart disease. ${ }^{31}$ Inflammation is implicated in the aetiology of these diseases, suggesting that there may be a somewhat general proinflammatory tendency among those with low resilience. The HRs for the association of stress resilience with IBD are of relatively small magnitude and this is consistent with our interpretation that stress does not initiate pathogenesis but may influence conversion from subclinical to frank symptomatic disease. While this suggests that stress may be implicated in disease progression, this study provides insufficient evidence to suggest that stress is of major clinical importance in determining IBD risk.

We considered a range of potential confounding factors. Socioeconomic and demographic factors in childhood and adolescence, like parental SEI, have previously been associated with IBD risk $^{32}$ and may be relevant to development of stress resilience. Since appendicitis before age 20 years has been associated with a reduced UC risk, ${ }^{10}$ we included this measure to increase precision when predicting risk. In this study, as previously observed, ${ }^{22}$ we saw evidence of subclinical disease activity in adolescence prior to IBD diagnosis in adulthood: higher inflammation level was associated with raised IBD risk, especially in CD, and low BMI was also associated with increased CD risk. Shorter stature in adolescence was associated with increased UC risk while overweight/obesity was associated with reduced risk. Lower BMI is often associated with malabsorption, especially in CD. Reduced growth rate in height can indicate the potential influence of malabsorption on growth in adolescence. ${ }^{33}$ We adjusted our analyses for these potential confounding factors (and excluded men with evidence of prodromal disease activity in a sensitivity analysis) as they might influence stress resilience. Adjustment for these factors or exclusion of men has a modest effect on the magnitude of the associations (the association with UC was enhanced in terms of statistical significance), and therefore it is less likely that stress resilience is being driven by early disease activity in adolescence. Further evidence that the direction of the association is from stress resilience to IBD is that the associations persisted after excluding men more likely to have subclinical IBD in adolescence and during the 15-year follow-up, even though this was at ages (2040 years) when IBD onset is more common ${ }^{9}$ and we had reduced statistical power.

There was no evidence of an interaction between stress resilience and the markers of prodromal disease activity with CD. However, low BMI was a risk for UC only among those with high stress resilience, suggesting that the apparent protective association of high stress resilience with UC is reduced in those with more aggressive subclinical disease in adolescence.

Our results for CD are consistent with the Nurses' Health Study, ${ }^{18}$ which looked at depressive symptoms and IBD risk and found these to be associated with a twofold increased CD risk. Unlike that study, we also found a statistically significant association with UC, of lower magnitude than we found for $\mathrm{CD}$. This may be due to statistical power as we had a larger number of participants. A case-control study with retrospectively reported stressful exposures ${ }^{19}$ and a cohort study of life events ${ }^{20}$ found no independent associations with IBD. The differences with our results could be due to a smaller number of events in the other studies, limiting power ${ }^{1920}$ or because they could not address individual variation in susceptibility to stress.

This cohort study had several practical advantages, including a prospectively collected measure of stress resilience before typical IBD onset age; we took into account potential effects of prodromal disease activity in adolescence and assessed stress resilience rather than major stressful events as a measure of psychosocial stress, since there are pronounced interindividual differences in susceptibility to stress. ${ }^{34}$ Some men were excluded due to missing conscription assessment data: since this 
tended to be for men unsuitable for military service (not all tests were required), the population, while broadly representative, would have excluded those in somewhat worse health.

Potential limitations include the lack of smoking information, as stress may increase the likelihood of heavier and prolonged smoking. ${ }^{35}$ Smoking is associated with increased CD risk and inversely associated with UC. ${ }^{5}$ Therefore, smoking might account for some of the association observed in CD (as a mediating mechanism), but cannot explain the observed raised UC risk associated with low stress resilience. Stress resilience was measured once during adolescence. Although we have seen longterm associations into middle age for this measure with future depression and anxiety, providing evidence that this measure is a stable and persistent characteristic, ${ }^{26}$ it is possible that resilience may change during adulthood, thus reducing the precision and possibly the magnitude of our estimates. Further potential limitations are that we do not have direct information on how stress is perceived, as captured by instruments such as the Perceived Stress Scale (PSS) $;{ }^{36}$ nor do we have direct information on coping strategies which are an important component of stressful experiences. ${ }^{36} 37$ Could some men with higher stress resilience have attempted to falsify their results towards lower resilience and therefore avoided some aspects of military service? We have no evidence of this but if this were the case, it would produce more conservative estimates. We have observed higher magnitude and consistent associations with other diseases, including depression and anxiety ${ }^{26}$ in subsequent adulthood, indicating that this is unlikely to be a major problem.

We were unable to take into account factors like diet, exposure to antibiotics and other personal characteristics that may be related to stress resilience and IBD risk (including 'triggering events', stressful or otherwise, that result in frank disease onset); thus, residual confounding is possible. We did adjust for parental SEI in childhood as this is relevant to the microbiological landscape during the window of susceptibility in early life. ${ }^{5}$ Patients with depression or anxiety (associated with low stress resilience $)^{26}$ may seek medical care frequently. ${ }^{38}$ Therefore, it is possible that those with lower stress resilience were more likely to seek medical care and therefore obtain an IBD diagnosis earlier as IBD diagnostic delay can be common among adults, ${ }^{39}$ and this could have influenced our results. However, the opposite has been shown to occur: individuals with low stress resilience can be reluctant to seek medical care, ${ }^{40}$ resulting in greater diagnostic delay. We know of no evidence to suggest that the genetic susceptibility to IBD influences stress resilience and if there were an association, our adjustment for markers of prodromal disease activity in adolescence would help to tackle confounding. The results apply to men up to age 57 years and may not be applicable to old ages or to women.

The positive predictive value (PPV), indicating the proportion of accurate records, is estimated as $85-95 \%$ for common diagnoses in the NPR. ${ }^{30}$ A recent validation of IBD diagnoses in the NPR, using more detailed and accurate information, reported a PPV of $90 \%$ for UC and $81 \%$ for CD for patients who did not subsequently change diagnosis between $\mathrm{UC}$ and $\mathrm{CD} .{ }^{41}$ Where such changes in diagnosis occurred, only $8 \%$ of UC and $6 \%$ of $\mathrm{CD}$ diagnoses were classified as non-IBD ${ }^{41}$ (change in IBD diagnosis only occurred in a minority of our study population). While we cannot rule out the possibility of some influence of error, this suggests that diagnostic inaccuracy is unlikely to account for our results, particularly as we performed sensitivity analyses to exclude the first 15 years of follow-up when diagnostic accuracy may have been less reliable due to less frequent use of endoscopy: this did not alter our results notably. The average age of IBD diagnosis indicates a slightly later onset than commonly reported, but the ages at diagnosis are consistent with other studies in Sweden conducted during the same time period. ${ }^{42}{ }^{43}$ In particular, patients with $\mathrm{CD}$ who had colorectal or small bowel disease were older at diagnosis. ${ }^{43}$

In summary, lower stress resilience in adolescence is associated with an increased risk of IBD diagnosis in adulthood, possibly through proinflammatory influences or reduction of intestinal barrier integrity.

Contributors SM and CM developed the hypothesis and design of the study. CM identified codes. CM prepared data and conducted analysis with support from $\mathrm{AH}$. SM, CM, KF and JH interpreted the study results. CM developed the first manuscript, and all authors edited critically and approved the final manuscript.

Funding Financial support was received from these sources: UK Economic and Social Research Council (ESRC) as grants to the International Centre for Life Course Studies (grant numbers RES-596-28-0001 and ES/J019119/1); Stiftelsen Olle Engkvist Byggmästare; and Strategic funding from Örebro University.

\section{Competing interests None declared.}

Ethics approval The Uppsala Regional Ethics Committee provided approval for the project (Dnr 2014/324). Ethical permission was given for access to data from national registers in a form where the identities of individuals could not be revealed to the researchers.

Provenance and peer review Not commissioned; externally peer reviewed.

Data sharing statement No additional data are available.

Open Access This is an Open Access article distributed in accordance with the Creative Commons Attribution Non Commercial (CC BY-NC 4.0) license, which permits others to distribute, remix, adapt, build upon this work noncommercially, and license their derivative works on different terms, provided the original work is properly cited and the use is non-commercial. See: http:// creativecommons.org/licenses/by-nc/4.0/

\section{REFERENCES}

1. Halfvarson J. Genetics in twins with Crohn's disease: less pronounced than previously believed? Inflamm Bowel Dis 2011;17:6-12

2. Jostins L, Ripke S, Weersma RK, et al. Host-microbe interactions have shaped the genetic architecture of inflammatory bowel disease. Nature 2012;491:119-24.

3. Gevers D, Kugathasan S, Denson LA, et al. The treatment-naive microbiome in new-onset Crohn's disease. Cell Host Microbe 2014;15:382-92.

4. Tysk C, Lindberg E, Jarnerot G, et al. Ulcerative colitis and Crohn's disease in an unselected population of monozygotic and dizygotic 
twins. A study of heritability and the influence of smoking. Gut 1988;29:990-6.

5. Loftus EV. Clinical epidemiology of inflammatory bowel disease: incidence, prevalence, and environmental influences. Gastroenterology 2004;126:1504-17.

6. Halfvarson J, Jess T, Magnuson A, et al. Environmental factors in inflammatory bowel disease: a co-twin control study of a Swedish-Danish twin population. Inflamm Bowel Dis 2006;12:925-33.

7. Hildebrand $\mathrm{H}$, Malmborg $\mathrm{P}$, Askling J, et al. Early-life exposures associated with antibiotic use and risk of subsequent Crohn's disease. Scand J Gastroenterol 2008;43:961-6.

8. Zhulina Y, Hahn-Strömberg V, Shamikh A, et al. Subclinical inflammation with increased neutrophil activity in healthy twin siblings reflect environmental influence in the pathogenesis of inflammatory bowel disease. Inflamm Bowel Dis 2013;19:1725-31.

9. Ruel J, Ruane D, Mehandru S, et al. IBD across the age spectrum: is it the same disease? Nat Rev Gastroenterol Hepatol 2014;11:88-98.

10. Andersson RE, Olaison G, Tysk C, et al. Appendectomy and protection against ulcerative colitis. N Engl J Med 2001;344: 808-14.

11. Nyboe Andersen N, Gørtz S, Frisch M, et al. Reduced risk of UC in families affected by appendicitis: a Danish national cohort study. Gut doi:10.1136/gutjnl-2015-311131 [Epub Ahead of Print $26 \mathrm{Apr}$ 2016].

12. Miller $\mathrm{AH}$, Maletic $\mathrm{V}$, Raison $\mathrm{CL}$. Inflammation and its discontents: the role of cytokines in the pathophysiology of major depression. Biol Psychiatry 2009;65:732-41.

13. de Punder K, Pruimboom L. Stress induces endotoxemia and low-grade inflammation by increasing barrier permeability. Front Immunol 2015;6:223.

14. Bitton A, Dobkin PL, Edwardes MD, et al. Predicting relapse in Crohn's disease: a biopsychosocial model. Gut 2008;57: 1386-92.

15. Bitton A, Sewitch MJ, Peppercorn MA, et al. Psychosocial determinants of relapse in ulcerative colitis: a longitudinal study. Am J Gastroenterol 2003;98:2203-8.

16. North CS, Alpers DH, Helzer JE, et al. Do life events or depression exacerbate inflammatory bowel disease? A prospective study. Ann Intern Med 1991;114:381-6.

17. von Wietersheim $\mathrm{J}$, Köhler $\mathrm{T}$, Feiereis $\mathrm{H}$. Relapse-precipitating life events and feelings in patients with inflammatory bowel disease. Psychother Psychosom 1992;58:103-12.

18. Ananthakrishnan AN, Khalili $\mathrm{H}$, Pan A, et al. Association between depressive symptoms and incidence of Crohn's disease and ulcerative colitis: results from the Nurses' Health Study. Clin Gastroenterol Hepatol 2013;11:57-62.

19. Lerebours E, Gower-Rousseau C, Merle V, et al. Stressful life events as a risk factor for inflammatory bowel disease onset: a population-based case-control study. Am J Gastroenterol 2007;102:122-31.

20. Li J, Norgard B, Precht DH, et al. Psychological stress and inflammatory bowel disease: a follow-up study in parents who lost a child in Denmark. Am J Gastroenterol 2004;99:1129-33.

21. Meaney MJ, Diorio J, Francis D, et al. Early environmental regulation of forebrain glucocorticoid receptor gene expression: implications for adrenocortical responses to stress. Dev Neurosci 1996;18:49-72

22. Melinder $\mathrm{C}$, Hiyoshi $\mathrm{A}$, Hussein $\mathrm{O}$, et al. Physical fitness in adolescence and subsequent inflammatory bowel disease risk. Clin Transl Gastroenterol 2015;6:e121.
23. Melinder C, Udumyan R, Hiyoshi A, et al. Decreased stress resilience in young men significantly increases the risk of subsequent peptic ulcer disease-a prospective study of 233093 men in Sweden. Aliment Pharmacol Ther 2015;41:1005-15.

24. Bergh C, Udumyan R, Fall K, et al. Stress resilience in male adolescents and subsequent stroke risk: cohort study. J Neurol Neurosurg Psychiatry 2014;85:1331-6.

25. Gunnarsson M, Udumyan R, Bahmanyar S, et al. Characteristics in childhood and adolescence associated with future multiple sclerosis risk in men: cohort study. Eur J Neurol 2015;22:1131-7.

26. Hiyoshi A, Udumyan R, Osika W, et al. Stress resilience in adolescence and subsequent antidepressant and anxiolytic medication in middle aged men: Swedish cohort study. Soc Sci Med 2015;134:43-9.

27. Stattin $\mathrm{H}$, Romelsjö A, Stenbacka M. Personal resources as modifiers of the risk for future criminality: an analysis of protective factors in relation to 18-year-old boys. $\mathrm{Br} J$ Criminol 1997;37:198-223.

28. Sorjonen K, Hemmingsson T, Lundin A, et al. Intelligence, socioeconomic background, emotional capacity, and level of education as predictors of attained socioeconomic position in a cohort of Swedish men. Intelligence 2012;40:269-77.

29. Otto U. Male youths. A sociopsychiatric study of a total annual population of Swedish adolescent boys. Acta Psychiatr Scand Suppl 1976;264:1-312.

30. Ludvigsson JF, Andersson E, Ekbom A, et al. External review and validation of the Swedish national inpatient register. BMC Public Health 2011;11:450.

31. Bergh $\mathrm{C}$, Udumyan $\mathrm{R}$, Fall $\mathrm{K}$, et al. Stress resilience and physical fitness in adolescence and risk of coronary heart disease in middle age. Heart 2015;101:623-9.

32. Gilat T, Hacohen D, Lilos $\mathrm{P}$, et al. Childhood factors in ulcerative colitis and Crohn's disease. An international cooperative study. Scand J Gastroenterol 1987;22:1009-24.

33. Van Gossum A, Cabre E, Hébuterne X, et al. ESPEN Guidelines on Parenteral Nutrition: gastroenterology. Clin Nutr 2009;28:415-27.

34. Shanks N, Larocque S, Meaney MJ. Neonatal endotoxin exposure alters the development of the hypothalamic-pituitary-adrenal axis: early illness and later responsivity to stress. J Neurosci 1995;15:376-84.

35. Jarvis MJ. Why people smoke. BMJ 2004;328:277-9.

36. Cohen S, Kamarck T, Mermelstein R. A global measure of perceived stress. J Health Soc Behav 1983;24:385-96.

37. Cohen S, Wills TA. Stress, social support, and the buffering hypothesis. Psychol Bull 1985;98:310-57.

38. Katon W, Lin EH, Kroenke K. The association of depression and anxiety with medical symptom burden in patients with chronic medical illness. Gen Hosp Psychiatry 2007;29:147-55.

39. Wagtmans MJ, Verspaget HW, Lamers CB, et al. Crohn's disease in the elderly: a comparison with young adults. J Clin Gastroenterol 1998;27:129-33.

40. Aday LA, Andersen R. A framework for the study of access to medical care. Health Serv Res 1974;9:208-20.

41. Jakobsson GL, Sternegård E, Olén $\mathrm{O}$, et al. Validating inflammatory bowel disease (IBD) in the Swedish National Patient Register and the Swedish Quality Register for IBD (SWIBREG). Scand J Gastroenterol 2017;52:216-21.

42. Ekbom A, Helmick C, Zack M, et al. The epidemiology of inflammatory bowel disease: a large, population-based study in Sweden. Gastroenterology 1991;100:350-8.

43. Lapidus A. Crohn's disease in Stockholm County during 1990-2001: an epidemiological update. World J Gastroenterol 2006;12:75-81. 